\title{
ON SEQUENTIAL CORES AND A THEOREM OF R. R. PHELPS
}

\author{
R. ATAlla AND J. BUSTOZ
}

1. Introduction. We will use the following notation. $A \backslash B$ means the set theoretic difference of $A$ and $B$, conv $B$ means the closed convex hull of $B$, and $\mathrm{Cl}_{B} A$ means the closure of $A$ in the set $B$. A sum $\sum$ without limits means $\sum_{1}^{\infty}$. The letters $s, t, r$ will be reserved to represent sequences $s=\left\{s_{n}\right\}, t=\left\{t_{n}\right\}$, etc.

Let $s=\left\{s_{n}(z)\right\}$ be a sequence of functions uniformly bounded in a domain $D$ of the complex plane. The Gibbs set of this sequence at $z_{0} \in \bar{D}$ is the set

$$
G\left(z_{0} ; s\right)=\left\{w: w=\lim _{k \rightarrow \infty} s_{n_{k}}\left(z_{k}\right), z_{k} \rightarrow z_{0}, n_{1}<n_{2}<\cdots\right\} .
$$

The set conv $G\left(z_{0} ; s\right)$ is called the core of the sequence at $z_{0}$. If $B$ is a regular matrix and $t=\left\{t_{n}(z)\right\}$ is the $B$-transform of $s=\left\{s_{n}(z)\right\}$ we will write $t=B s$. The following theorem relates the core of $s$ to the core of $B s$.

THEOREM 1. The relation conv $G\left(z_{0} ; B s\right)$ Conv $G\left(z_{0} ; s\right)$ holds for every uniformly bounded $\left\{s_{n}(z)\right\}$ if and only if

$$
\lim \sup (n \rightarrow \infty) \sum\left|b_{n k}\right|=1 .
$$

Theorem 1 was proved by Agnew [1] for sequences of complex numbers. The stated extension to sequences of functions is given in [3]. This theorem bears a similarity to a theorem proved by Phelps [4]. We will state this last theorem below.

Let $X$ and $Y$ be sets, and let $A$ and $B$ be linear spaces of complex valued bounded functions on $X$ and $Y$ respectively. It is assumed that both $A$ and $B$ contain the constant functions and are normed with sup norm. Phelps's theorem follows.

TheOREM 2. Let $T$ be a linear operator from $A$ to $B$. Then $\|T\|=1$ and $T(1)=1$ if and only if $(T f)(Y) \subset \operatorname{conv} f(X)$ for every $f \in A$. The operator $T$ is an isometry if and only if $\operatorname{conv}(T f)(Y)=\operatorname{conv} f(X)$ for each $f \in A$.

The main result of the present paper is that Theorem 1 is a consequence of Theorem 2. In establishing this result we will show that the Banach algebra $A$ consisting of suitably defined equivalence

Received by the editors May 14, 1968. 
classes of uniformly bounded sequences is isometrically isomorphic to $C(X)$ for a certain compact space $X$. Limiting values of sequences are then the same as the values of corresponding functions on $X$.

For a regular matrix $B$, the operator norm of $B$ as an operator on $A$ is given by (1.01). That is,

$$
\|B\|=\lim \sup (n \rightarrow \infty) \sum\left|b_{n k}\right| \text {. }
$$

The norm of the induced operator $B^{\prime}$ on $C(X)$ is equal to $\|B\|$. Also, regularity of $B$ implies that $B^{\prime}(1)=1$. Thus the matrix $B$ of Theorem 1 satisfies the hypothesis of Theorem 2 .

The authors are grateful to the referee for suggestions on shortening and simplifying $\$ 3$.

2. The Banach algebra of uniformly bounded sequences. Let $D$ be a completely regular topological space and $C(D)$ the class of complex valued, bounded, continuous functions on $D$. We will write $\|f\|_{1}$ for the sup norm on $C(D)$. Let $A^{\prime}$ be the class of sequences $s=\left\{s_{n}\right\}, s_{n} \in C(D)$, with $\lim \sup (n \rightarrow \infty)\left\|s_{n}\right\|_{1}<\infty . A^{\prime}$ is a complex algebra under the operations

$$
s+t=\left\{s_{n}+t_{n}\right\}, \quad \alpha s=\left\{\alpha s_{n}\right\}, \quad s t=\left\{s_{n} t_{n}\right\} .
$$

$A^{\prime}$ becomes a pseudo-normed Banach algebra with $\|s\|$ $=\lim \sup (n \rightarrow \infty)\left\|s_{n}\right\|_{1}$. We define equivalence classes in $A^{\prime}$ by $s \sim t$ if and only if $\|s-t\|=0$. Clearly $s \sim t$ if and only if $\left\{s_{n}-t_{n}\right\}$ converges uniformly to zero. We define a norm in the space $A$ of equivalence classes thus formed by $\|x\|_{2}=\{\|s\|:$ any $s \in x\}$. Then $A$ is a Banach algebra.

For a regular matrix $B=\left(b_{n k}\right)$ and an equivalence class $x \in A$ we define $B x=\{t: t=B s, s \in x\}$. It is not hard to show that $B x \in A$. Hence $B$ is an operator from $A$ into $A$.

The next theorem shows that $A$ with \|\|$_{2}$ gives the proper form to the operator norm $\|B\|_{2}$. For convenience we will write

$$
\beta=\lim \sup (n \rightarrow \infty) \sum\left|b_{n k}\right| \text {. }
$$

TheOREM 3. $\|B\|_{2}=\beta$.

Proof. Let $x$ be an equivalence class in $A$ and $s=\left\{s_{n}\right\} \in x$. Then

$$
\begin{aligned}
\|B x\|_{2} & =\lim \sup (n \rightarrow \infty)\left\|\sum b_{n k} s_{k}\right\|_{1} \\
& \leqq \lim \sup (n \rightarrow \infty) \sum\left|b_{n k}\right|\left\|s_{k}\right\|_{1} .
\end{aligned}
$$

Given $\epsilon>0$ there exists $K=K(\epsilon)$ such that $k \geqq K$ implies $\left\|s_{k}\right\|_{1} \leqq \epsilon$ $+\|s\|_{2}$. Then 


$$
\|B s\|_{2} \leqq \lim \sup (n \rightarrow \infty) \sum_{k=1}^{K}\left|b_{n k}\right|\left\|s_{k}\right\|_{1}+\left[\epsilon+\|s\|_{2}\right] \cdot \beta
$$

The first term on the right of this last inequality is zero. Hence $\|B s\|_{2} \leqq \beta\|s\|_{2}$ and $\|B\|_{2} \leqq \beta$.

Suppose now that $\|B\|_{2}<\beta$. There exists a sequence of indices $\{n(p)\}$ and a number $\lambda>0$ such that

$$
\sum\left|b_{n(p), k}\right|-\|B\|>\lambda ; \quad p=1,2, \cdots .
$$

Then for any sequence $s=\left\{s_{k}\right\}$ with $\|s\|_{2}=1$ we must have

$$
\sum\left|b_{n(p), k}\right|-\|B s\|>\lambda ; \quad p=1,2, \cdots .
$$

For a given $p$ define $s$ by $s_{k}=\exp \left(-i \arg b_{n(p), k}\right)$. Then clearly $\|s\|_{2}=1$, but $s$ violates (1.03). Hence $\|B\|<\beta$ is impossible and we have $\|B\|=\beta$.

It may be noted that only the conditions that the columns go to zero and the rows are a bounded subset of $l^{1}$ are needed. For such matrices it is easy to check that the equivalence class decompositions we have introduced preserve convergence fields, that is, if $\left\|B-B^{\prime}\right\|_{2}$ $=0$, then $C_{B}=C_{B^{\prime}}$, and if $s, t$ are sequences such that $\|s-t\|=0$, then $s \in C_{B}$ if and only if $t \in C_{B}$. This fact is not needed below.

3. Representation of $A$ as $C(X), X$ compact. Let $Y$ be a locally compact space, $C(Y)$ the bounded continuous complex valued function on $Y$, and $\operatorname{Co}(Y)$ the elements of $(Y)$ which disappear at infinity. $\operatorname{Co}(Y)$ consists exactly of the elements of $C(Y)$ whose extensions to $\beta Y$ vanish on $\beta Y \backslash Y$ (cf. [4], exercise $7 \mathrm{~F} .1$ ). It is easy to see that the quotient space $C(Y) / \mathrm{Co}(Y)$ may be mapped isomorphically into $C(\beta Y \backslash Y)$. Since $Y$ is locally compact, $\beta Y \backslash Y$ is compact, hence $C^{*}$-embedded in $\beta Y$, and it follows that the isomorphism is onto.

Letting $D$ be as in $\S 2$, we may regard each element of $C(D)$ as defined on $\beta D$. If $N$ is the positive integers, $N \times \beta D$ is locally compact, and it follows that the algebra $A$ is isomorphic to $C(X)$, where $X=\beta(N \times \beta D) \backslash(N \times \beta D)$. The isomorphism is actually an isometry, for if $s=\left\{s_{n}\right\} \in A^{\prime}$ and $\bar{s}$ is the restriction to $X$ of its extension to $\beta(N \times \beta D)$, then $\|s\|=\lim \sup \left\|s_{n}\right\|$. This follows from the important fact that if $\Omega \in X$ and $\left\{\left(n_{\alpha}, w_{\alpha}\right)\right\}$ is a net in $N \times \beta D$ such that $\left(n_{\alpha}, w_{\alpha}\right)$ $\rightarrow \Omega$, then $n_{\alpha} \rightarrow \infty$ in $N$.

Finally we note that a regular matrix $B$ such that $\|B\|=1$ induces an operator $B^{\prime}$ on $C(X)$ defined by $B^{\prime}(\bar{s})=\overline{B s}$. The operator $B^{\prime}$ satisfies $\left\|B^{\prime}\right\|=1$ and $B^{\prime} 1=1$. That $\left\|B^{\prime}\right\|=1$ follows from Theorem 3 . To show $B^{\prime} 1=1$, let $x \in A$ and suppose $\bar{s}(\Omega)=1$ for all $\Omega \in X$ and $s=\left\{s_{n}\right\} \in X$. If $\left(n_{\alpha}, w_{\alpha}\right)$ is a net in $N \times D$ and $\left(n_{\alpha}, w_{\alpha}\right) \rightarrow \Omega \in X$, then 
$s\left(n_{\alpha}, w_{\alpha}\right) \rightarrow 1$. If $t=B s$, regularity of $B$ gives $t\left(n_{\alpha}, w_{\alpha}\right) \rightarrow 1$, so $\bar{t}(\Omega)=1$ for all $\Omega \in X$.

From now on we will simply write $B$ for $B^{\prime}$ and it will be clear from the context whether $B$ is acting on $A$ or $C(X)$.

4. The core theorem. We assume now that $D$ is a subset of the complex plane with the discrete topology. For $z_{0} \in \bar{D}$ define the sets $B_{n}\left(z_{0}\right)=\left\{z \in D:\left|z-z_{0}\right|<1 / n\right\}$ and $K=\bigcap_{n=1}^{\infty} \mathrm{Cl}_{X}\left(N \times B_{n}\left(z_{0}\right)\right)$.

TheOREM 4. $\bar{s}(K)=G\left(z_{0} ; s\right)$.

Proof. We first show that the left side is contained in the right side. Let $t \in \bar{s}(K)$. Then $t=\bar{s}(\Omega), \Omega \in K$. Define the sets $V_{n}$ $=\left\{\Omega^{\prime} \in \beta(N \times \beta D):\left|\bar{s}\left(\Omega^{\prime}\right)-t\right|<1 / n\right\}$. For every $n$ we have $\Omega \in \mathrm{Cl}_{X}\left(N \times B_{n}\left(z_{0}\right)\right)$ and hence there exists $m(n) \geqq n$ and $z_{n} \in B_{n}\left(z_{0}\right)$ such that $\Omega^{\prime}=\left(m(n), z_{n}\right) \in V_{n}$. Thus $1 / n>\left|\bar{s}\left(\Omega^{\prime}\right)-t\right|=\left|s_{m(n)}\left(z_{n}\right)-t\right|$ so that $t \in G\left(z_{0}, s\right)$.

For the reverse inclusion let $t \in G\left(z_{0}, s\right)$. Then $t=\lim s_{n(k)}\left(z_{k}\right)$ with $z_{n} \rightarrow z_{0}, n(1)<n(2)<\cdots$. We may then write $z_{k} \in B_{m(k)}\left(z_{0}\right)$ with $m(1)<m(2)<\cdots$. Let $\Omega \in X$ be a cluster point of $\left(n(k), z_{k}\right)$ so that $\bar{s}(\Omega)=t$. To show that $\Omega \in \mathrm{Cl}_{X}\left(N \times B_{n}\left(z_{0}\right)\right)$ note that for some $k, m(k)$ $\geqq n$ and so $B_{m(k)}\left(z_{0}\right) \subset B_{n}\left(z_{0}\right)$. Then $j \geqq k$ implies $\left(n_{j}, z_{j}\right) \in N \times B_{n}\left(z_{0}\right)$. Thus $\Omega$ is a cluster point of $N \times B_{n}\left(z_{0}\right)$.

We will state the core theorem again and prove it by applying Theorem 2.

TheOREM 5. Let $B=\left(b_{n k}\right)$ be a regular matrix. Then $\|B\|=1$ if and only if $G\left(z_{0} ; B s\right) \subset$ conv $G\left(z_{0} ; s\right)$ for every $s \in A^{\prime}$ and every $z_{0} \in \bar{D}$.

Proof. Let $K$ be defined as above. For $N, n=1,2, \cdots$, let $t_{n}^{N}(z)=1$ if $z \in B_{N}\left(z_{0}\right)$ and $t_{n}^{N}(z)=0$ if $z \notin B_{N-1}\left(z_{0}\right)$. Then $t_{n}^{N}(\Omega)=1$ if $\Omega \in K_{N}$ and $t_{n}^{N}(\Omega)=0$ if $\Omega \notin K_{N-1}$, where $K_{n}=\mathrm{Cl}_{X}\left[N \times B_{n}\left(z_{0}\right)\right]$, $n$ $=1,2, \cdots$.

Set $\left\{r_{n}^{N}\right\}=\left\{s_{n} t_{n}^{N}\right\}$ and let $r_{N} \in C(X)$ correspond to $\left\{r_{n}^{N}\right\} . r_{N}(\Omega)=1$ if $\Omega \in K_{N}$ and $r_{N}(\Omega)=0$ if $\Omega \notin K_{N-1}$. Then $\sum b_{n k} r_{k}^{N}(z)=\sum b_{n k} s_{k}(z)$ if $z \in K_{N}$ and $\sum b_{n \mathbf{z}} r_{\boldsymbol{k}}^{N}(z)=0$ if $z \notin K_{N-1}$. Hence $B r_{N}(\Omega)=B s(\Omega)$ if $\Omega \in K_{N}$ and $B r_{N}(\Omega)=0$ if $\Omega \notin K_{N-1}$.

By Theorem $2 \mathrm{Br}_{N}(X) \subset$ conv $r_{N}(X)$. We may assume without loss of generality that $0 \in s(K)$. Using the fact that $K_{N}$ compact and $f$ continuous imply $\bigcap_{N} f\left(K_{N}\right)=f\left(\bigcap_{N} K_{N}\right)$ we have:

$$
\begin{aligned}
s(K) & =\bigcap_{N=1}^{\infty} B r_{N}\left(K_{N}\right) \subseteq \bigcap_{N=1}^{\infty} B r_{N}(X) \subseteq \bigcap_{N=1}^{\infty} \operatorname{conv} r_{N}(X) \\
& =\operatorname{conv} \bigcap_{N=1}^{\infty} r_{N}(X)=\operatorname{conv} \bigcap_{N=1}^{\infty} r_{N}\left(K_{N-1}\right)=\operatorname{conv} s(X) .
\end{aligned}
$$


Hence $G\left(z_{0} ; B s\right) \subseteq \operatorname{conv} G\left(z_{0} ; s\right)$.

To prove the converse we note that by Theorem 2 if $\|B\| \neq 1$ then there exists $s \in C(X)$ such that $B s(X) \subseteq \bar{s}(X)$. Let $t \in B s(X) \backslash s(X)$. Clearly $t \notin G\left(z_{0} ; s\right)$ for any $z_{0} \in \bar{D}$. We will show that $t \in G\left(z_{0} ; B s\right)$ for some $z_{0} \in \bar{D}$.

There exists $\Omega \in X$ such that $B s(\Omega)=t$. Also, there is a net $\left(n_{\alpha}, z_{\alpha}\right)$ in $N \times D$ such that $\left(n_{\alpha}, z_{\alpha}\right) \rightarrow \Omega$ and $n_{\alpha} \rightarrow \infty$ in $N$. Then $\lim t_{n(\alpha)}\left(z_{\alpha}\right)=t$. Since $n_{\alpha} \rightarrow \infty$ in $N$, and $s_{n(\alpha)}\left(z_{\alpha}\right) \rightarrow t$, there exists a sequence $\left\{m_{j}\right\}$ $\subset\left\{n_{j}\right\}$ such that $m_{1}<m_{2}<\cdots$, and if $z_{j}$ are the corresponding $z_{\alpha}$ 's, we have $s_{m(j)}\left(z_{j}\right) \rightarrow t$. If $z_{0}$ is a cluster point of $\left\{z_{j}\right\}$, then there are suitable subsequences $\left\{p_{j}\right\} \subset\left\{m_{j}\right\}$ and $\left\{x_{j}\right\} \subset\left\{z_{j}\right\}, p_{1}<p_{2}<\cdots$, $x_{j} \rightarrow z_{0}$ such that $\lim t_{p(j)}\left(x_{j}\right)=t$. Hence $t \in G\left(z_{0}, B s\right)$.

The second part of Theorem 2 concerning the isometry $B$, can also be applied to the core theorem. This gives

TheOREM 6. conv $G\left(z_{0} ; B s\right)=\operatorname{conv} G\left(z_{0} ; s\right)$ if and only if the regular matrix $B$ is an isometry on $A$.

It has been shown by H. S. Allen [2] that a regular matrix $B=\left(b_{n k}\right)$ leaves the core of every bounded sequence of complex numbers invariant if and only if

(a) $\|B\|=1$.

(b) For every sequence of suffixes $P(i)(i=1,2, \cdots)$ the number 1 is a limit of the sequence

$$
U_{n}=\sum a_{n, P(i)}
$$

We can restate Allen's result as

Theorem 7. If $D$ is a one point space so that $A^{\prime}$ consists of bounded sequences of complex numbers then the regular matrix $B$ is an isometry on $A$ if and only if $B$ satisfies conditions (a) and (b) above.

THEOREM 8. Let $A^{\prime}$ be the class of sequences of functions uniformly bounded in the unit disc. Then the regular matrix $B$ leaves cores invariant and hence is an isometry on $A$ if and only if

(i) $\|B\|=1$.

(ii) For every sequence of suffixes $n(1)<n(2)<\cdots$ there exists a subsequence of $\{n(i)\}$ say $p(1)<p(2)<\cdots$ and a sequence of suffixes $m(1)<m(2)<\cdots$ such that $\lim \sup (k \rightarrow \infty) b_{m(k), p(k)}=1$.

Proof. Suppose first that $B$ satisfies the stated conditions. Then for every $s \in A$ by Theorem 5 we have conv $G\left(z_{0} ; B s\right) \subseteq \operatorname{conv} G\left(z_{0} ; s\right)$. We must prove the reverse inclusion.

Let $w \in G\left(z_{0} ; s\right)$. Then $w=\lim s_{n(k)}\left(z_{k}\right)$ where $n(1)<n(2)<\cdots$ and $z_{k} \rightarrow z_{0}$. Let $p(1)<p(2)<\cdots$ be the subsequence of $\{n(i)\}$ and 
$m(1)<m(2)<\cdots$ the sequence as defined in (ii). Denote by $\left\{\zeta_{j}\right\}$ the subsequence of $\left\{z_{k}\right\}$ corresponding to $\{p(j)\}$. Then $s_{p(j)}\left(\zeta_{j}\right) \rightarrow w$. For the transform of $s$ we have

$$
t_{m(r)}\left(\zeta_{r}\right)=b_{m(r), p(r)} s_{p(r)}\left(\zeta_{r}\right)+\sum^{\prime} b_{m(r), n} s_{n}\left(z_{n}\right)
$$

where the prime on the sum indicates that $n=p(r)$ is omitted.

Now since lim $\sup (r \rightarrow \infty)\left|b_{m(r), p(r)}\right|=1$ it follows from (i) that

$$
\lim \sup (r \rightarrow \infty)\left|\sum^{\prime} b_{m(r), n} s_{n}\left(z_{n}\right)\right|=0 .
$$

Hence $t_{m(r)}\left(\zeta_{r}\right) \rightarrow w$ and $w \in G\left(z_{0} ; B s\right)$. Clearly then conv $G\left(z_{0} ; B s\right)$ $\supseteq$ conv $G\left(z_{0} ; s\right)$ and the sufficiency of (i) and (ii) is proved.

The necessity of (i) follows from Theorem 5 . To prove that (ii) is also necessary we assume the existence of a sequence of suffixes $\{n(i)\}$ that violates (ii). That is, $\lim \sup \left|b_{m(r), p(r)}\right|<1$ for every subsequence $\{p(j)\}$ of $\{n(i)\}$ and every sequence of suffixes $m(1)$ $<m(2)<\cdots$. Using a diagonalization argument it can be shown that there must then exist $\alpha, 0<\alpha<1$ such that

$$
\lim \sup (r \rightarrow \infty)\left|b_{m(r), p(r)}\right|<\alpha
$$

for every pair of sequences $m(r)$ and $p(r)$ as defined above.

We now define the sequence of functions $s=\left\{s_{n}(x)\right\}$ as follows.

$$
\begin{aligned}
& s_{n}(x) \equiv 0 \quad \text { if } n \notin\{n(i)\}, \\
& s_{n(i)}(x)=1 \quad x=1 / n(i) \text {, } \\
& =0 \quad x \neq 1 / n(i) .
\end{aligned}
$$

It is obvious that conv $G(0 ; s)=[0,1]$. For any sequence $m(1)$ $<m(2)<\cdots$ we have

$$
t_{m(r)}(x)=\sum b_{m(r), n} s_{n}(x)=\sum b_{m(r), n(i)} s_{n(i)}(x) .
$$

Clearly $t_{m(r)}(x)=0$ whenever $x$ is not of the form $1 / n(j)$ and hence $0 \in G(0 ; A s)$. Let $\left\{x_{r}\right\}$ be any sequence with $x_{r} \rightarrow 0$ and consider $t_{m(r)}\left(x_{r}\right)$. Again it is clear that $t_{m(r)}\left(x_{r}\right)=0$ unless $x_{r}=1 / Q(r)$ where $Q(r) \in\{n(i)\}$. Then $t_{m(r)}\left(x_{r}\right)=0$ or $t_{m(r)}\left(x_{r}\right)=b_{m(r), Q(r)}$. But

$$
\lim \sup (r \rightarrow \infty)\left|b_{m(r), Q(r)}\right|<\alpha<1
$$

and hence conv $G(0 ; A s) \neq$ conv $G(0 ; s)$. Thus condition (ii) is necessary. This completes the proof.

It is easily seen that any matrix satisfying (i) and (ii) of Theorem 8 must also satisfy (a) and (b) of Theorem 7. The authors do not know if the converse is true. It is of interest in this connection to note that the examples given in [2] of matrices satisfying (a) and (b) are matrices that satisfy (i) and (ii). 
According to Hoffman [5, p. 69] an isometry of $C(X)$ onto $C(X)$ is multiplicative, i.e. $T(f g)=T(f) T(g)$. It follows that the matrix operators discussed in Theorems 6,7 , and 8 are multiplicative at infinity in a natural sense.

As a final remark, we note that the proofs of Theorems 4, 5, 6, and 8 go through with little modification if $K$ is replaced by a normal topological space, and $\left\{B_{n}\right\}$ is a sequence of open sets with closure such that $\mathrm{Cl} B_{n+1} \subset B_{n}$.

\section{REFERENCES}

1. R. P. Agnew, Cores of complex sequences and of their transforms, Amer. J. Math. 61 (1939), 178-186.

2. H. S. Allen, T-transformations which leave the core of every bounded sequence invariant, J. London Math. Soc. 19 (1944), 42-46.

3. J. Bustoz, Gibbs sets and the generalized Gibbs phenomenon, Dissertation, Arizona State University, 1967.

4. L. Gilman and M. Jerison, Rings of continuous functions, Van Nostrand, New York, 1960.

5. K. Hoffman, Fundamentals of Banach algebras, Universidade do Paraná, Curitiba, Rio de Janeiro, 1962.

6. R. R. Phelps, The range of Tf for certain linear operators $T$, Proc. Amer. Math. Soc. 16 (1965), 381-382.

OHIO UNIVERSITY 